\title{
Carrier-mediated Translocation of Uridine Diphosphate Glucose into the Lumen of Endoplasmic Reticulum-derived Vesicles from Rat Liver
}

\author{
Florent Vanstapel and Norbert Blanckaert \\ Departments of Laboratory Medicine and Medicine, and the Liver Center, University of California School of Medicine, San Francisco, \\ California 94143-0134
}

\begin{abstract}
Radiolabeled UDPGlc incubated with rough endoplasmic reticulum (RER)-derived microsomes from rat liver became associated with the vesicles. This microsomal uptake of nucleotide sugar was time and temperature dependent. Analysis of the molecular species containing radiolabel revealed that initial uptake represented entry of predominantly intact UDPGIc in the microsomes. Conclusive evidence for proper translocation of UDPGlc across the microsomal membrane into the intravesicular space was obtained by demonstrating that UDPGlc was transported into an osmotically sensitive compartment. Microsomal uptake of UDPGlc exhibited features characteristic of carrier-mediated transport including saturation, specificity, and countertransport. Inhibition and transstimulation studies showed that other uridine-containing nucleotide sugars and $5^{\prime}$-UMP were substrates of the postulated microsomal carrier system for UDPGlc, while cytosine- or guanosine-containing nucleotides and non-5'-uridine monophosphates were, at best, very poor substrates. UDPGlc translocation activities were lower in smooth microsomal fractions than in the RER-derived vesicles, indicating that contamination with Golgi membranes could not be responsible for microsomal transport of UDPGlc. Our findings suggest that rat liver endoplasmic reticulum possesses a carrier system mediating proper translocation of UDPGlc and 5'-uridine-substituted structural analogues across the membrane.
\end{abstract}

\section{Introduction}

A family of hepatic microsomal UDP-glycosyltransferases play a crucial role in detoxification, inactivation, and/or efficient excretion of a vast array of xenobiotics and various endogenous substances including bilirubin, steroids, catecholamines, and thyroid hormone (1). The glycosyl moiety transferred to these substrates is derived from nucleotide sugars synthesized in the cytoplasm. Because there is remarkable latency of the glycosyltransferase activities in native, sealed liver microsomes, it has been suggested that it is necessary to transport the charged, membrane-impermeant nucleotide sugars across the endoplasmic reticulum (ER) ${ }^{1}$ membrane to reach the active

Address reprint requests to Dr. Blanckaert, Clinical Pathology, UZ KUL Medical Center, B-3000 Leuven, Belgium.

Received for publication 28 May 1987 and in revised form 8 February 1988

1. Abbreviations used in this paper: Chapso, 3-[(3-cholamido-propyl) dimethyl ammonio]-2-hydroxy-1-propane-sulfonate; ER, endoplasmic reticulum; RER, rough endoplasmic reticulum.

J. Clin. Invest.

(C) The American Society for Clinical Investigation, Inc. $0021-9738 / 88 / 09 / 1113 / 10 \$ 2.00$

Volume 82, September 1988, 1113-1122 center of the enzyme (2). Similar latency of enzymic activity is found with the microsomal glycosyltransferases which catalyze the synthesis of the (GlcNAc) ${ }_{2}-P$ - $P$-Dol and Glc- $P$-Dol precursors of the oligosaccharide- $P$ - $P$-Dol serving as donor substrate in cotranslational $\mathrm{N}$-glycosylation of proteins in the ER (3). However, no direct evidence was available that UDPGlcNAc, UDPGlc, or the cosubstrates of the UDP-glycosyltransferase system (UDPGIcUA, UDPGlc, UDPXyl) are transported across the ER membrane, and current knowledge of the topology of the transferases in the transverse plane of the membrane is fragmentary and inconclusive. In fact, it has been suggested that the active center of UDP-glucuronyltransferase faces the cytoplasmic side of the ER and that the latency of the enzyme activity may reflect the presence of the transferase in a "constrained" conformational form in the microsomal membrane $(4,5)$.

We recently presented evidence that native intact microsomes from rat liver contain an intravesicular pool of endogenous UDPGlc (6). Because UDPGlc is synthesized by cytosolic enzymes, this finding raised the question whether the intramicrosomal UDPGlc pool reflected presence of UDPGlc within the cisternal space of the ER of the intact cell and therefore reflected the existence of a mechanism for transport of UDPGlc across the ER membrane, or was artifactually formed by incorporation of cytosolic fluid occurring when the ER membranes are transformed to vesicles during homogenization. Because we found that the intramicrosomal UDPGlc pool could be enlarged and an intravesicular pool of UDPXyl could be established by preincubating intact microsomes with the corresponding exogenous nucleotide sugar (6), transport of UDPGlc and UDPXyl across the microsomal membrane permeability barrier seemed to be a plausible possibility. Moreover, such transport appeared to be highly temperature dependent since the preloading effect was markedly decreased when preincubation of the vesicles with exogenous UDPGlc or UDPXyl was performed at $0^{\circ} \mathrm{C}$ instead of at $37^{\circ} \mathrm{C}$. Perez and Hirschberg (7) recently reported on carrier-mediated microsomal uptake of UDPGlc but failed to demonstrate translocation of the intact nucleotide sugar across the membrane into the lumenal space of the vesicles and existence of a pool of intact UDPGlc in the microsomes.

The following questions were explicitly addressed in the studies reported here. (a) Does intact UDPGlc traverse the permeability barrier of the ER membrane in intact microsomal vesicles? (b) Does uptake of intact UDPGlc by microsomes correspond either to entry into the intravesicular space or to formation of a membraneous pool of UDPGlc and/or its metabolites? $(c)$ Is such membrane translocation of UDPGlc mediated by a carrier system for nucleotide sugars in the ER membrane? Our results suggest that a carrier system mediating translocation of UDPGlc across the membrane into the intramicrosomal space is present in RER-derived vesicles. These findings have major implications for glycosylation reactions catalyzed by microsomal UDP-glycosyltransferases and glyco- 
syltransferases that are involved in synthesis of the Dol-P-P-(GlcNAc) ${ }_{2} \mathrm{Man}_{9} \mathrm{Glc}_{3}$ oligosaccharide donor for cotranslational core $N$-glycosylation of nascent polypeptides.

\section{Methods}

Chemicals. All unlabeled nucleotides, bovine serum albumin fraction V, D-mannose(Man)-6-P,4-(2-hydroxy-ethyl)-1-piperazine ethanesulfonic acid (Hepes), raffinose, and ovomucoid were purchased from Sigma Chemical Co. (St. Louis, MO). 3-[(3-cholamido-propyl)dimethyl ammonio]-2-hydroxy-1-propane-sulfonate (Chapso) was from Bio-Rad Laboratories (Richmond, CA). UDP $\left[{ }^{3} \mathrm{H}\right] \mathrm{Glc}(385 \mathrm{~Bq} / \mathrm{pmol}$ ) and $\left[{ }^{14} \mathrm{C}\right]$ sucrose $(24.9 \mathrm{~Bq} / \mathrm{pmol})$ were purchased from New England Nuclear (Boston, MA). [ $\left.{ }^{14} \mathrm{C}\right]$ UMP (11.1 Bq/pmol) was from ICN (Irvine, CA).

General procedures. The following analytes or enzymatic activities were assayed as described in the corresponding references: total protein using bovine serum albumin as calibration standard (8), Man-6-phosphatase (9), RNA (10), UDP-galactosyltransferase using ovomucoid as acceptor substrate (11), NADPH-cytochrome $c$ reductase (12).

Animals and preparation and treatments of microsomes. Male Wistar-derived inbred R/A Pfd rats (13) weighing 220-280 g were killed by decapitation after an overnight fast of 20-22 h. A $25 \%$ (wt/ vol) liver homogenate was made in ice-cold $0.25 \mathrm{M}$ sucrose by 20 strokes with a loose-fitting pestle in a Dounce glass homogenizer. Postmitochondrial supernatant was obtained by centrifugation of the homogenate at $41,000 \mathrm{~g}$ for $7 \mathrm{~min}$ at $4^{\circ} \mathrm{C}$ (Sorvall SS-34 rotor, 18,500 $\mathrm{rpm}$ ). Rough ER membrane-derived vesicles (RER microsomes) and smooth microsomes were prepared by the procedure of Eriksson et al. (14). Postmitochondrial supernatant $(7 \mathrm{ml})$ was layered on top of 2.5 $\mathrm{ml}$ of $0.60 \mathrm{M}$ sucrose which in turn was layered on $7 \mathrm{ml}$ of $1.30 \mathrm{M}$ sucrose. Both sucrose layers contained $15 \mathrm{mM} \mathrm{CsCl}$. Ultracentrifugation $\left(105,000 \mathrm{~g}\right.$ for $90 \mathrm{~min}$ at $4^{\circ} \mathrm{C}$; Beckman Ti-60 or Ti-50.2 rotor) resulted in selective pelleting of RER microsomes and accumulation of a smooth fraction around the interface between the $0.60 \mathrm{M}$ and $1.30 \mathrm{M}$ sucrose layers. The RER pellet was resuspended in $\sim 7 \mathrm{ml}$ of $0.25 \mathrm{M}$ sucrose that contained $1.0 \mathrm{mM} \mathrm{Na}_{2}$ EDTA and was buffered to $\mathrm{pH} 7.4$ with $5 \mathrm{mM}$ Tris/ $\mathrm{HCl}$ (buffer A). RER microsomes were subsequently repelleted $\left(105,000 \mathrm{~g}\right.$ for $30 \mathrm{~min}$ at $\left.4^{\circ} \mathrm{C}\right)$. Washing with the EDTAcontaining buffer removed about $65 \%$ of the RNA present in the first RER pellet. The smooth microsomal fraction was diluted threefold with buffer $A$ before repelleting. Before washing the membrane preparations with buffer A, RER vesicles were 4.2- to 5-fold enriched in RNA in comparison with smooth microsomes. Washed and resuspended RER and smooth microsomes ( $\sim 40 \mathrm{mg}$ protein $/ \mathrm{ml}$ ) were stored under an argon atmosphere at $-70^{\circ} \mathrm{C}$ for up to $1 \mathrm{mo}$. Structural intactness of the microsomes was routinely verified by determination of Man-6-phosphatase latency (15), which was $>95 \%$ in all preparations used. "Fully disrupted microsomes" refers to membrane preparations that had been pretreated for $30-60$ min at $0^{\circ} \mathrm{C}$ with $4 \mathrm{mM}$ Chapso. Such treatment resulted in complete elimination of latency of Man-6-phosphatase (9). Specific activities of the Golgi marker UDPGal/ovomucoid galactosyl-transferase in RER membrane preparations was $32.0 \pm 11.3 \mathrm{pmoles} / \mathrm{mg}$ protein per $\min (n=16)$, which amounted to $\sim 58 \%$ (range $42-67 \% ; n=10$ ) and $\sim 22 \%$ (range: $16-28 \% ; n=6$ ) of the specific activities observed in homogenates and smooth microsomes, respectively. Transmission electron microscopy of the membrane preparations before washing with the EDTA-containing buffer A confirmed that the RER microsomes corresponded to vesicular structures with ribosomes on their cytoplasmic side and negligible contamination with Golgi membranes. Smooth microsomal preparations had a similar vesicular appearance but were devoid of ribosomes and contained relatively few Golgi membrane profiles.

Translocation assay. Uptake of radiolabeled nucleotide or sucrose into microsomes was determined by a rapid filtration technique (16, 17) using mixed cellulose filters (type HAWP $02500,0.45 \mu \mathrm{m}$ pore size, from Millipore Corp., Bedford, MA) and an ultrafiltration manifold (FH 225V; Hoefer Scientific Instruments, San Francisco, CA). Microsomes (10 mg protein/ml except when stated otherwise) were resuspended in argon-flushed ice-cold $0.25 \mathrm{M}$ sucrose containing 10 $\mathrm{mM} \mathrm{NaF}$ and buffered to $\mathrm{pH} 7.4$ with $10 \mathrm{mM}$ Hepes (buffer B). To inhibit microsomal nucleotide pyrophosphatase (18), 2,3-dimercaptopropanol $(5 \mathrm{mM})$, and $\alpha, \beta$-methylene adenosine $5^{\prime}$-triphosphate (1 $\mathrm{mM}$ ) were added to this microsomal suspension immediately before starting incubation at $37^{\circ} \mathrm{C}$. Buffer $\mathrm{B}$ with these two additional ingredients is denoted as "incubation buffer." The mixture was then blanketed with argon and brought to the temperature selected for assaying translocation $\left(37^{\circ} \mathrm{C}\right.$ except when stated otherwise).

When solute translocation was assessed by influx measurement, seven volumes of microsomal suspension were mixed with one volume of buffer B containing the radiolabeled test solute ("start solution") to initiate the uptake reaction. At various time intervals, aliquots (usually $20 \mu \mathrm{l}$ ) containing about $20,000 \mathrm{cpm}$ of ${ }^{3} \mathrm{H}$ - or ${ }^{14} \mathrm{C}$-radioactivity were removed from the incubation mixture and immediately mixed with 1 ml buffer $\mathrm{C}$ (buffer B containing $16.25 \mathrm{mM} \mathrm{MgCl}$ ) to arrest uptake. Presence of $\mathrm{MgCl}_{2}$ in buffer $\mathrm{C}$ was required to obtain retention of the microsomes by the filters. Without delay, vesicle-associated radiolabel was separated from free, extramicrosomal radiolabel by rapid filtration of $900 \mu \mathrm{l}$ of the mixture, followed by immediate washing of the retentate on the filter with $5 \mathrm{ml}$ ice-cold stop solution. Filtration and rinsing were completed within 15-20 s. Washing with more of the stop solution did not change the radioactivity retained on the filter. The filter was placed in a scintillation vial, dissolved in $1 \mathrm{ml}$ scintillation grade 2-methoxyethanol, and counted in a Packard (model 4530) liquid scintillation counter; (Packard Instruments Co., Downers Grove, IL) after adding $10 \mathrm{ml}$ of Scinti-Verse II (Fisher Scientific, Pittsburgh, PA) scintillator fluid.

When translocation was assessed by efflux measurement, microsomal vesicles were first preloaded by preincubation at $37^{\circ} \mathrm{C}$ with radioactive nucleotide sugar in incubation buffer. In a second step, efflux of radiolabel was measured after abrupt 20-fold dilution of the microsomal suspension in buffer C. Aliquots of $400 \mu \mathrm{l}$ were withdrawn at 30-s intervals and immediately pipetted onto the Millipore filters for separation of microsomal label from extramicrosomal label by rapid filtration. Washing of the filter and counting of retained radioactivity were done as described for the uptake assays.

Adsorption of radioactive UDPGlc to the filters was determined by replacing the microsomal suspension in the assay mixture with incubation buffer and was found to be negligible (retained radioactivity $<0.01 \%$ of radiolabel applied to filter). Nonspecific binding of the radiolabeled test solute to microsomes and filter was routinely assessed by mixing the microsomal suspension and the radiolabel-containing start solution at $0^{\circ} \mathrm{C}$, immediately adding $20 \mu$ of this mixture to $1 \mathrm{ml}$ of buffer $\mathrm{C}$, and determining radioactivity in the retentate on the Millipore filter when $900 \mu \mathrm{l}$ of this blank sample was submitted to the rapid ultrafiltration procedure. It constituted $<0.05 \%$ of the radioactivity applied to the filter. Identical results were obtained when both the microsomal suspension and the start solution were incubated separately for $60 \mathrm{~min}$ at $37^{\circ} \mathrm{C}$ before mixing at $0^{\circ} \mathrm{C}$ of these two components with buffer $\mathrm{C}$. These blank values were at least one order of magnitude lower than the uptake values obtained with test samples that had been incubated at $37^{\circ} \mathrm{C}$. Such blank values were routinely determined and subtracted from values measured with test specimens for computation of uptake values. Because considerable metabolism of the radiolabeled test nucleotides taken up in the microsomes may occur, vesicle-associated radioactivity is expressed as radiolabeled nucleotide equivalents. Initial rates of microsomal uptake of radiolabel were assessed by determining vesicle-associated radioactivity at five time points during the initial $1.5 \mathrm{~min}$ after mixing of microsomes with radiolabeled substrate. The initial uptake rate was estimated by calculation of the second degree polynomial equation fitting the uptake values as a function of incubation time.

Loss of microsomal protein in the ultrafiltrate was $<5 \%$ of the 
protein applied to the filter, as assessed by measuring $A_{280 \mathrm{~mm}}$ and assaying NADPH-cytochrome $c$ reductase. RER microsomes remained $>93 \%$ intact, as determined by assay of the latency of Man-6-phosphatase, during translocation assay incubations at $37^{\circ} \mathrm{C}$ for up to $60 \mathrm{~min}$.

Analysis of radiolabeled product formed from nucleotide sugars. Microsomal transformation of radiolabeled UDP-sugar to protein- or lipid-linked derivatives during incubation was assessed by measurement of radioactivity in TCA-precipitable and lipid-extractable compounds using methods described by Godelaine et al. (19). The composition of the soluble radioactive components present in RER microsomes that had taken up UDP $\left[{ }^{3} \mathrm{H}\right] \mathrm{Glc}$ was determined as follows. Vesicle-associated radiolabel was separated from extramicrosomal radioactivity by the rapid filtration method. After washing the filter with $5 \mathrm{ml}$ of buffer $\mathrm{C}$, additional washing was performed with $4 \mathrm{ml}$ deionized water containing unlabeled UDPGlc, Glc-1-P, and free Glc (200 $\mathrm{nmol}$ of each). The eluate was mixed with $2 \mathrm{ml}$ ethanol. After evaporation in vacuo of the azeotropic solvent, the residue was redissolved in an ethanol/water (3:2; vol/vol) mixture for application to a silica gel plate (60F-254, 5765, $0.25 \mathrm{~mm}$; EM Laboratories, Elmsford, NY). The plates were developed using $n$-butanol/acetone/glacial acetic acid/ concentrated ammonium hydroxide/water (25:25:9:0.75:40; by vol) as solvent system (20). The bands migrating as UDPGlc, Glc-1-P, and free Glc in the chromatogram were visualized by $254 \mathrm{~nm}$ ultravioletlight and spraying with naphthoresorcinol reagent and identified by cochromatography of the individual reference compounds on both sides of the sample. The bands were then scraped into scintillation vials for counting of radioactivity. Hydrolysis was undetectable when the radiolabeled UDP-sugar was directly subjected to the concentration and chromatography procedures

\section{Results}

Time- and temperature-dependent microsomal uptake of UDPGlc. Time curves for uptake of radiolabeled by RER microsomes incubated at $37^{\circ} \mathrm{C}$ with $25 \mu \mathrm{M}$ or $2 \mathrm{mM} \mathrm{UDP}\left[{ }^{3} \mathrm{H}\right]-$ Glc are shown in Fig. 1. The initial rate of microsomal uptake of radiolabeled solute was lower at decreased temperatures and dropped to $<5 \%$ of the initial rate found at $37^{\circ} \mathrm{C}$ when incubation was performed at $0^{\circ} \mathrm{C}$, indicating that uptake reflected a highly temperature-dependent process. Based on the Arrhenius plot shown in Fig. 2, the energy of activation for the uptake reaction was estimated at $86 \mathrm{~kJ} / \mathrm{mol}$. Comparable temperature dependency of microsomal uptake rate was observed with low and high UDPGlc substrate concentrations. The radiolabel uptake rate at $0^{\circ} \mathrm{C}$ found with $25 \mu \mathrm{M}$ and $2 \mathrm{mM}$ $\mathrm{UDP}\left[{ }^{3} \mathrm{H}\right]$ Glc averaged, respectively, $2.1 \%(n=3)$ and $3.2 \%(n$ $=2$ ) of the rate at $37^{\circ} \mathrm{C}$. At the $25 \mu \mathrm{M}$ as well as at the $2 \mathrm{mM}$ $\mathrm{UDP}\left[{ }^{3} \mathrm{H}\right] \mathrm{Glc}$ concentration, initial uptake rates were directly proportional to microsomal protein concentration in the incubation medium ranging from 2 to $10 \mathrm{mg}$ protein $/ \mathrm{ml}$.

Microsomal uptake of ${ }^{3} \mathrm{H}$-label at low UDPGlc concentrations ( $<50 \mu \mathrm{M})$ consistently exhibited an initial "overshoot" phase during which radioactivity in the microsomes transiently exceeded the amount of radiolabel present in the vesicles at the latest time point, when uptake equilibrium of UDPGlc was approached (Fig. $1 A$ ). Microsomes incubated with $25 \mu \mathrm{M}$ UDPGlc contained $242 \pm 85$ (mean \pm SD; $n=6$ ) pmol labeled UDPGlc equivalents/mg protein when overshooting peaked, after $10 \mathrm{~min}$. In a subsequent phase, net loss of radionuclide from the microsomes occurred resulting in apparent equilibration of UDPGlc between the extramicrosomal medium and microsomal vesicles. This biphasic uptake

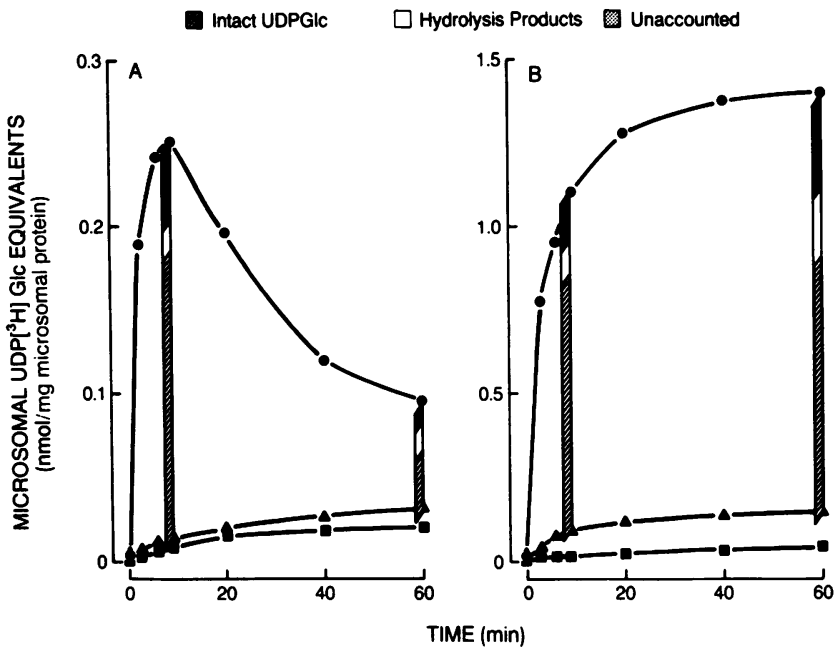

Figure 1. Microsomal uptake and metabolism of UDPGlc. RER microsomes were incubated at $37^{\circ} \mathrm{C}$ with either $25 \mathrm{mM}(A)$ or $2 \mathrm{mM}$ UDP $\left[{ }^{3} \mathrm{H}\right] \mathrm{Glc}$. Uptake of total radioactivity in the vesicles (๑) was measured by the rapid filtration method. Concurrent formation of nonsoluble metabolites was assessed by determination of lipid-extractable ( $\square$ ) and TCA-precipitable ( $\Delta$ ) radioactivity in the microsomes. The composition of the soluble metabolites released by hypotonic shock of the vesicles was determined after 9 and $60 \mathrm{~min}$ of incubation and is presented in the bar diagrams superimposed on the time curves. The fraction denoted as "unaccounted" corresponds to the radioactivity that was associated with the vesicles but was unaccounted for in the TCA-precipitable radiolabeled metabolites and radiolabel recovered from the thin-layer chromatogram.

process usually was lost when the substrate concentration exceeded $400 \mu \mathrm{M}$. The evidence that the overshoot phenomenon probably reflects trans-stimulation of UDPGlc uptake by en-

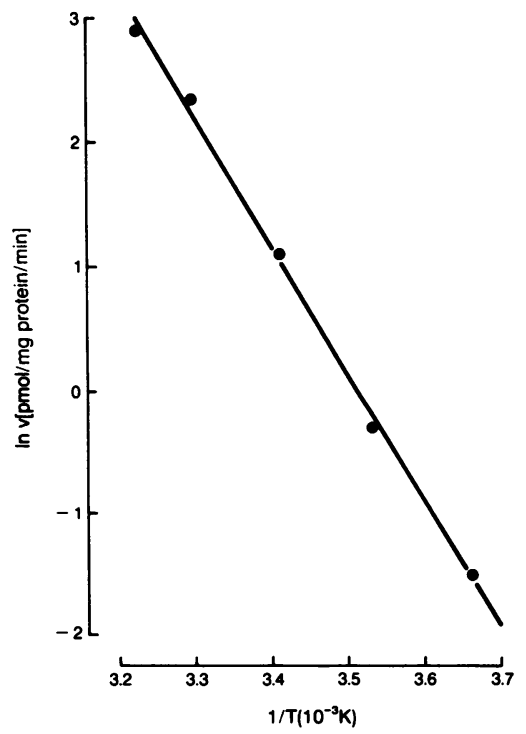

Figure 2. Temperature dependence of microsomal uptake of UDPGlc. RER-derived microsomes were first preincubated for 40 min at $37^{\circ} \mathrm{C}$. Portions of the incubation mixture were then brought separately to either $37^{\circ} \mathrm{C}, 30^{\circ} \mathrm{C}, 20^{\circ} \mathrm{C}$, $10^{\circ} \mathrm{C}$, or $0^{\circ} \mathrm{C}$. After thermal equilibration was reached, $\left[{ }^{3} \mathrm{H}\right] \mathrm{Glc}$ substrate $(25 \mu \mathrm{M})$ was added and initial uptake of radiolabel by the vesicles $(11 \mathrm{mg}$ protein/ $\mathrm{ml}$ ) was determined with the rapid filtration method. Linear regression analysis on the results presented in an Arrhenius plot gave a slope corresponding to an energy of activation of $86 \mathrm{~kJ} / \mathrm{mol}\left(r^{2}\right.$ $=0.997$ ). 
dogenous solutes contained in the microsomes will be presented below.

Metabolism of UDPGlc taken up by microsomes. To determine the extent to which radiolabel associated with the microsomes reflected authentic UDPGlc, we performed fractionation of the radiolabeled molecular species in a TCA-precipitable portion (representing protein-linked and lipid-linked species), a lipid-extractable portion (lipid-linked species), and a soluble fraction that was not precipitated by treatment of the specimen with TCA. Incubation of microsomes at $37^{\circ} \mathrm{C}$ with $25 \mu \mathrm{M}$ or $2 \mathrm{mM}$ UDP $\left[{ }^{3} \mathrm{H}\right] \mathrm{Glc}$ resulted in gradual formation of lipid-extractable and TCA-precipitable radiolabeled compounds (Fig. 1). Neither of these solutes was detectable in the filtrates. Microsomal formation of the two fractions accounted for only a minor portion of the radioactive solutes in the microsomes. With $25 \mu \mathrm{M}$ UDPGlc substrate, TCA-precipitable material comprised $<10 \%(6.4 \pm 1.3 \%, n=3)$ of the total microsomal radiolabel after $10 \mathrm{~min}$ of incubation. Rates of microsomal formation of nonsoluble metabolites rapidly fell as a function of incubation time. The TCA-precipitable fraction of the microsomal radiolabel measured after $60 \mathrm{~min}$ of incubation, when equilibrium uptake was reached or approached, gradually decreased as a function of UDPGlc substrate concentration, from $38.2 \pm 7.0 \%(n=4)$ at $25 \mu \mathrm{M}$ UDPGlc to $13.8 \pm 5.5 \%(n=2)$ at $2 \mathrm{mM}$ UDPGlc.

To determine the composition of the soluble radioactive substances present in the vesicles, washed microsomes retained on the filter were subjected to hypotonic shock in order to release lumenal solutes. The released soluble compounds were subsequently subjected to thin-layer chromatography using unlabeled UDPGlc, Glc-1-P, and free Glc as reference, and the ${ }^{3} \mathrm{H}$-label migrating with each of these three compounds was analyzed. Total radioactivity recovered in the three bands accounted for $80.8 \pm 5.6 \%(n=9)$ of the total soluble (i.e., not precipitable by TCA) solutes present in the microsomes before the hypotonic shock treatment. For RER microsomes incubated during $10 \mathrm{~min}$ at $37^{\circ} \mathrm{C}$ with $25 \mu \mathrm{M}$ UDPGlc, intact UDPGlc amounted to $93 \%$ of the total radiolabel in the chromatogram. Under the reasonable assumption that this composition was similar in the small fraction of microsomal radioactivity that was not extracted from the microsomes and/or not recovered from the chromatogram, our findings suggest that authentic UDPGlc accounted for about $87 \%$ of the total radionuclide taken up by the microsomes after 10 min of incubation. When equilibrium uptake was approached after $60 \mathrm{~min}$ of incubation, the fraction of microsome-associated radiolabel recovered in the hydrolytic products, Glc-1-P and Glc, had risen to $32 \%$ and $18 \%$ when tested with, respectively, $25 \mu \mathrm{M}$ and $2 \mathrm{mM}$ UDPGlc substrate.

Collectively, our results indicated that during the initial phase of the time curves, before equilibrium uptake was reached and especially during the overshoot phase observed at low UDPGlc substrate concentrations, radiolabel that became associated with the RER microsomes was present in authentic UDPGlc. This finding strongly suggested that most, if not all, radiolabel entered the microsomes in the form of intact nucleotide sugar, with subsequent metabolism to hydrolytic products and possibly also to lipid- and protein-linked derivatives.

Evidence that UDPGlc enters the lumen of the microsomal vesicles. The finding that most of the microsomal radiolabel was released when the vesicles were subjected to hypotonic shock indicated that UDPGlc had entered the microsomal lumen. In addition, the osmotic sensitivity of UDPGlc uptake by the vesicle membrane preparations from RER was determined in order to distinguish between membrane binding and proper translocation. Intravesicular lumenal volume can be modified by the addition of varying concentrations of an impermeant solute to the incubation medium. Under these circumstances, amounts of radiolabeled solutes taken up by the microsomes at equilibrium uptake should directly reflect changes in intravesicular volume if the label enters into the internal space of the vesicles. Fig. 3 shows the effect of increasing osmolarity of the incubation medium on UDPGlc and sucrose equilibrium uptake by RER microsomes. That the chosen impermeant solute raffinose was osmotically competent was evidenced by the gradual decrease of the sucrose-accessible space of the microsomes as a function of the trisac-

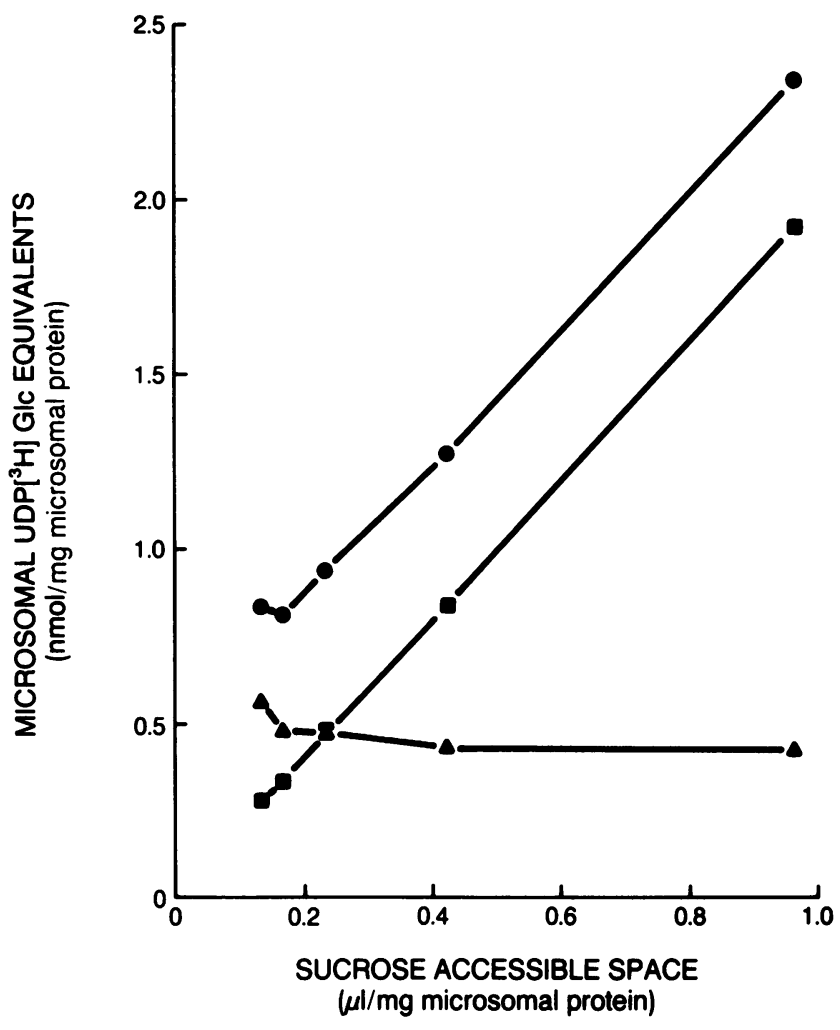

Figure 3. Effect of incubation medium osmolarity on UDPGlc- and sucrose-accessible space in RER microsomes. Microsomes were preincubated for $15 \mathrm{~min}$ at $37^{\circ} \mathrm{C}$ in incubation buffer containing various concentrations of raffinose $(0,100,200,333,500 \mathrm{mM})$. Both UDP $\left[{ }^{3} \mathrm{H}\right] \mathrm{Glc}(2 \mathrm{mM})$ and $\left[{ }^{14} \mathrm{C}\right]$ sucrose were added, and vesicle-associated radioactivity was determined by the rapid filtration method after $30 \mathrm{~min}$ and $45 \mathrm{~min}$ of incubation to verify that equilibration uptake of the radioactive solutes was reached at the end of the incubation period. The depicted results are those found at $45 \mathrm{~min}$. Volumes of space accessible to radiolabel in the microsomes were computed according to Meissner and Allen (11). Increase of the raffinose concentration from zero to $0.5 \mathrm{M}$ resulted in shrinkage of the sucrose-accessible space from 0.963 to $0.139 \mu \mathrm{l} / \mathrm{mg}$ protein. The figure depicts the corresponding volumes for apparent space accessed by

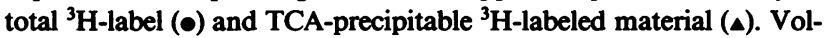
umes for the apparent space occupied by the soluble ${ }^{3} \mathrm{H}$-labeled solutes ( $\square$ ), which predominantly corresponded to intact UDPGlc, were computed by subtracting the TCA-precipitable fraction from total vesicle-associated ${ }^{3} \mathrm{H}$-label. 
charide concentration in the incubation medium. This shrinkage of the vesicles was paralleled by a proportional decrease of the total amount of UDPGlc-derived radiolabel present in the microsomes at equilibrium uptake (at $45 \mathrm{~min}$ ). A high UDPGlc substrate concentration ( $2 \mathrm{mM}$ ) was selected for these experiments to avoid the overshoot phenomenon and thereby to ascertain that equilibrium uptake was reached at the end of the 45-min incubation period. An additional advantage of using such high substrate levels was that even after $45 \mathrm{~min}$ of incubation only a small fraction of the vesicle-associated radioactivity was converted to hydrolysis products of UDPGlc, so that the ${ }^{3} \mathrm{H}$-label of the soluble radioactive solutes in the microsomes approximated the amount of the radiolabeled authentic UDPGIc (Fig. $1 B$ ). The amount of nonsoluble, TCAprecipitable solutes containing ${ }^{3} \mathrm{H}$-label derived from UDPGlc and found in the microsomes at equilibrium uptake was unaffected by the addition of raffinose and therefore was relatively important in the vesicle preparations that had undergone marked shrinkage, as a result of exposure to high raffinose concentrations (Fig. 3). By subtraction of the TCA-precipitable fraction from total radionuclide in the microsomes, we computed the amount of authentic nucleotide sugar at UDPGlc equilibrium uptake. Fig. 3 shows that there was a fair linear relationship between the intravesicular volume of the microsomes, as measured by the sucrose space, and the microsomal space occupied at equilibrium uptake by UDPGlc, as estimated by measuring the amount of soluble radiolabeled material.

Additional information pertinent to distinction between membrane binding and proper translocation can be obtainedby extrapolation to zero sucrose-accessible space. Since at this point the intravesicular space would be zero, the amount of vesicle-associated radiolabel is thought to represent membrane binding at equilibrium uptake. It is noteworthy that the line describing the relationship between sucrose space and the computed space for soluble radiolabeled material intersected near to the origin (Fig. 3), which indicates that nonspecific membrane binding of UDPGlc and its hydrolysis products was negligible. This finding confirmed the very low values for nonspecific binding of intact UDPGlc found by direct measurement (see Methods).

Collectively, these findings provided convincing evidence for the notion that temperature-dependent microsomal uptake of UDPGlc corresponded to translocation across the membrane, with entry of the nucleotide sugar into the lumenal space of the vesicles.

Trans-stimulation of translocation of UDPGlc across the microsomal membrane. Based on the typical shape of the time curves for microsomal uptake of radiolabel at low UDPGlc substrate concentrations (Fig. $1 \mathrm{~A}$ ), we considered the possibility that the aforementioned overshoot phenomenon reflected trans-stimulation caused by countertransport of endogenous unlabeled solute(s) sharing a putative carrier with UDPGlc for transport across the membrane. This idea was consistent with our previous finding that native microsomes contain a lumenal pool of endogenous UDPGlc (6) and was supported by the results obtained in the following three experimental approaches. First, we found that preincubation of native microsomes at $37^{\circ} \mathrm{C}$, which produced depletion of the microsomal pool of endogenous UDPGlc (6), resulted in a gradual decrease of the overshoot phenomenon (Fig. $4 \mathrm{~A}$ ). With most microsomal preparations, overshooting was abolished when prein-
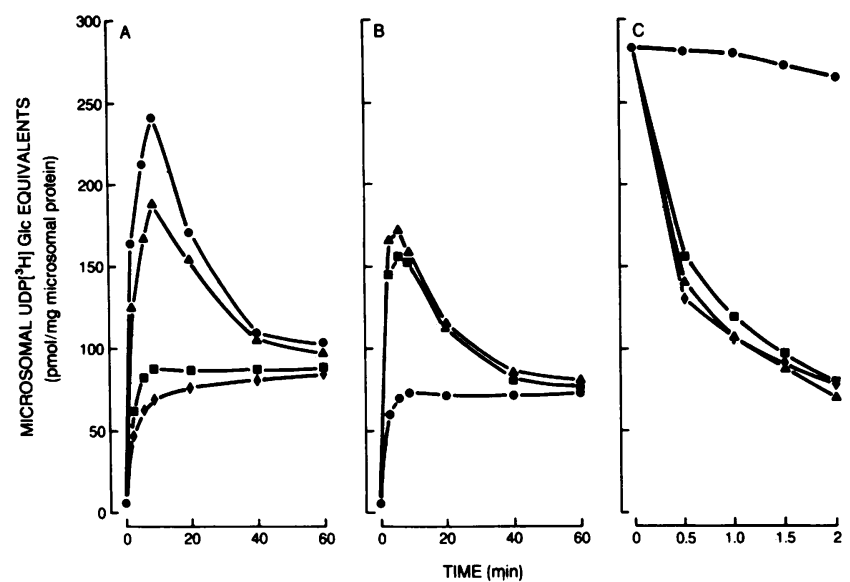

Figure 4. Trans-stimulation of microsomal translocation of UDPGlc. RER-derived microsomes were employed in all experiments, and 25 $\mu \mathrm{M}$ UDP $\left[{ }^{3} \mathrm{H}\right] \mathrm{Glc}$ was used as initial substrate concentration in the translocation assays. $(A)$ Time curves for uptake of ${ }^{3} \mathrm{H}$-label by vesicles that has been preincubated at $37^{\circ} \mathrm{C}$ in incubation buffer for zero $\min (\bullet), 3 \mathrm{~min}(\Delta), 10 \mathrm{~min}(\bullet)$, or $15 \mathrm{~min}(\bullet)$ before the radiolabeled substrate was added. $(B)$ The effect of preloading the microsomes

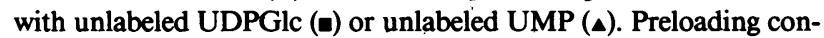
sisted of preincubation at $37^{\circ} \mathrm{C}$ for $15 \mathrm{~min}$ of the microsomes $(12 \mathrm{mg}$ protein $/ \mathrm{ml}$ ) in incubation buffer without (controls, $\bullet$ ) or with $5 \mathrm{mM}$ UDPGlc or UMP. The microsomes were then washed twice, each time by resuspending the vesicles in ice-cold buffer $A(\sim 0.6 \mathrm{mg}$ protein $/ \mathrm{ml}$ ) and repelleting at $105,000 \mathrm{~g}$ for $30 \mathrm{~min}$ at $4^{\circ} \mathrm{C}$. $(C)$ Vesicles preloaded by preincubation for $10 \mathrm{~min}$ at $37^{\circ} \mathrm{C}$ in incubation buffer with $25 \mu \mathrm{M}$ UDP $\left[{ }^{3} \mathrm{H}\right] \mathrm{Glc}$ were used. At time zero, the microsomes were 20 -fold diluted by adding buffer $\mathrm{C}$ without (controls, $\bullet$ ) or with

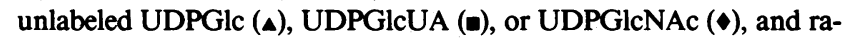
diolabeled efflux from the vesicles was measured as described in Methods. The concentration of unlabeled trans-stimulating nucleotide in the translocation assay mixture was $200 \mu \mathrm{M}$.

cubation at $37^{\circ} \mathrm{C}$ had been extended beyond $30 \mathrm{~min}$. Contrariwise, overshooting remained virtually unaffected when preincubation of native microsomes was done at $0^{\circ} \mathrm{C}$ for as long as $1 \mathrm{~h}$, which did not lead to depletion of the endogenous UDPGlc in native microsomes (6). Using a second appioach (Fig. $4 \mathrm{~B}$ ), we found that marked trans-stimulation of microsomal uptake of radiolabeled UDPGle could be produced by preloading the vesicles with either unlabeled UDPGlc or unlabeled UMP, which may also be present in native rat liver microsomes (21). In a third, complementary approach; we demonstrated that addition of unlabeled UDPGlc, UDPGlcUA, or UDPGlcNAc to the extravesicular incubation :medium markedly enhanced the initial efflux rate of radiolabel from RER microsomes preloaded with radiolabeled UDPGIc (Fig. $4 C$ ), suggesting that massive net influx of unlabelęd UDPGlc or its structural analogues caused trans-stimulation of membrane translocation of the radiolabeled UDPGlc.

The notion that the gvershoot phenomenon reflected countertransport in which influx of radiolabeled solute at low UDPGlc substrate concentrations was accelerated by a large gradient across the microsomal membrane of unlabeled endogenous "driver" solute(s) like UDPGlc itself or structural analogues was further supported by the observation of a comparable transient overshoot when microsomal uptake of $\left[{ }^{14} \mathrm{C}\right]-$ UMP by native RER microsomes was tested (Fig. $5 \mathrm{~A}$ ). Complementary findings consistent with the concept of membrane 


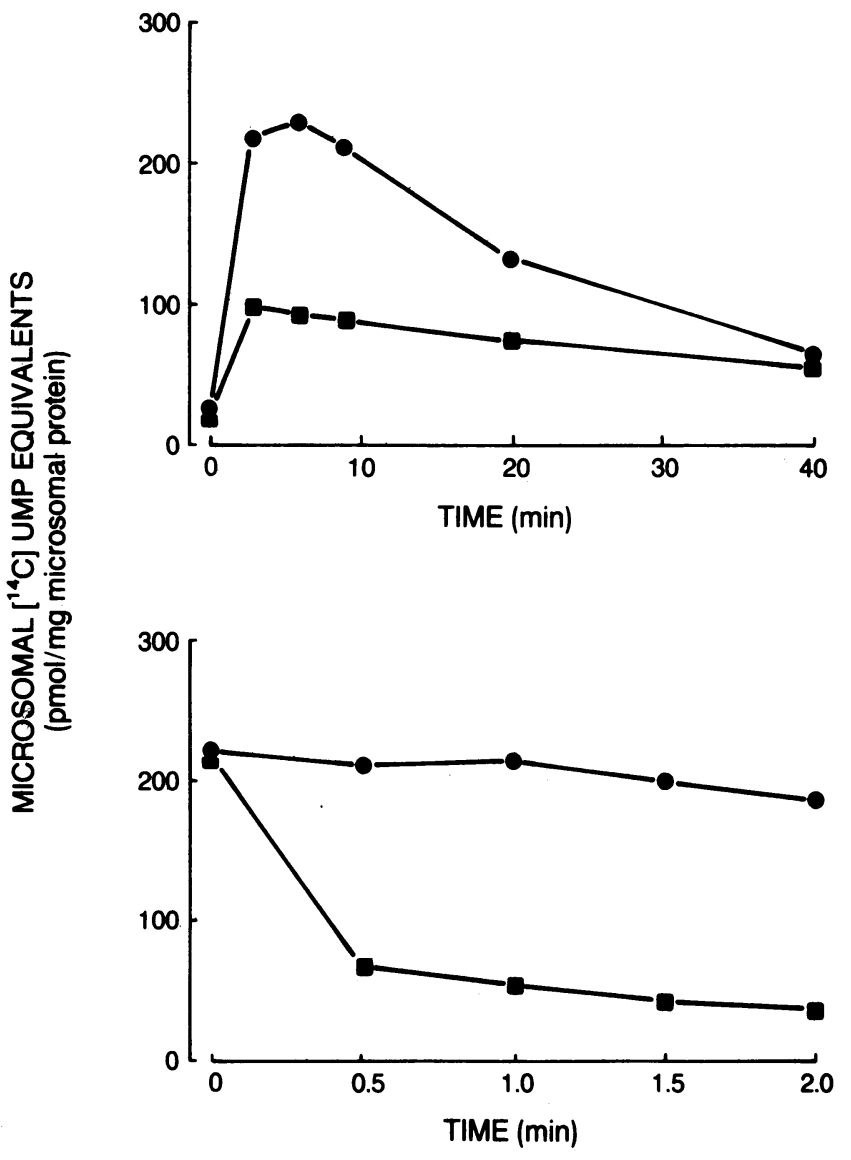

Figure 5. Effects of UDPGlc on microsomal translocation of UMP. RER microsomes that were kept on ice until the uptake reaction was started by addition of $25 \mu \mathrm{M}\left[{ }^{14} \mathrm{C}\right] \mathrm{U}$ MP substrate were used in both depicted experiments. Uptake of ${ }^{14} \mathrm{C}$-label by microsomes was measured with ( $\bullet$ ) and without (๑) $200 \mu \mathrm{M}$ unlabeled UDPGlc present in the incubation mixture (upper panel). Measurement of UMP efflux in the presence ( $($ ) and absence ( $\bullet$ ) of $200 \mu \mathrm{M}$ unlabeled UDPGlc was done as described in the legend to Fig. $4 C$ except that $25 \mu \mathrm{M}$ $\left[{ }^{14} \mathrm{C}\right]$ UMP substrate was used to preload the microsomes.

translocation of UDPGlc and UMP mediated by a common microsomal carrier system consisted of demonstration that addition of unlabeled UDPGlc to the incubation medium caused marked cis-inhibition of microsomal UMP uptake (Fig. $5 \mathrm{~A}$ ) and trans-stimulation of UMP efflux from preloaded vesicles (Fig. 5 B).

Specificity of microsomal translocation of nucleotides. The specificity of the postulated microsomal carrier system for UDPGlc was examined by the addition of structural analogues to the incubation media. Each of the tested 5'-uridine nucleotides (UDPGicUA, UDPGal, UDPGlcNAc, UDPXyl, UMP) added in eightfold excess over the radiolabeled UDPGlc substrate effectively cis-inhibited microsomal uptake of radiolabel (Table I). In contrast, cytidine- and guanosine-containing sugar nucleotides or monophosphates either were markedly less effective cis-inhibitors or exerted no significant effect on UDPGlc uptake. Strong additional support for specificity of the putative carrier translocating UDPGlc came from the observation that a mixture of 2 '-UMP and 3 '-UMP lacked any effect on uptake while the 5'-UMP isomer clearly was an effec-
Table I. Inhibition of Microsomal UDPGlc Uptake by Structurally Related Molecules

\begin{tabular}{lc}
\hline Tested compound & UDPGlc uptake rate \\
\hline & $\%$ control \\
UDPGlc & $40 \pm 2.2$ \\
UDPXyl & $45 \pm 3.9$ \\
UDPGlcUA & $57 \pm 1.3$ \\
UDPGal & $59 \pm 1.5$ \\
UDPGlcNAc & $50 \pm 3.8$ \\
5'-UMP $^{\prime}$-UMP, 3'-UMP & $52 \pm 1.9$ \\
CMP & $100 \pm 1.3$ \\
GMP & $82 \pm 1.6$ \\
CMPNeuAc & $86 \pm 4.5$ \\
CDPChol & $95 \pm 2.9$ \\
GDPMan & $90 \pm 3.2$ \\
GDPGlc & $90 \pm 0.2$ \\
& $98 \pm 3.7$ \\
\hline
\end{tabular}

After preincubation of RER-derived microsomes for $40 \mathrm{~min}$ at $37^{\circ} \mathrm{C}$, UDP $\left[{ }^{3} \mathrm{H}\right]$ Glc $(25 \mu \mathrm{M})$ with or without unlabeled inhibitor $(200 \mu \mathrm{M})$ was added. Initial rate of radiolabel uptake by the vesicles $(10 \mathrm{mg}$ protein $/ \mathrm{ml}$ ) was then measured in four aliquots of the reaction mixture, sampled at 20-s time intervals. Data shown are mean values \pm SEM for three individual experiments using different microsomal preparations.

tive cis-inhibitor (Table I) and trans-stimulator (Fig. $4 \mathrm{~B}$ ) of membrane translocation of UDPGlc. Free Glc and GlcUA and also their respective 1-monophosphate derivatives tested at both 8-fold and 40-fold molar excess over the radiolabeled UDPGlc substrate concentration ( $25 \mu \mathrm{M})$ equally lacked any appreciable effect on microsomal uptake of radiolabel.

Dependence of rate of microsomal uptake of UDPGlc on substrate concentration. To distinguish between passive equilibration and facilitated transport of UDPGlc, the effect of substrate concentration on initial rate of uptake was investigated. In view of the interference with initial rate measurements at low substrate concentration by endogenous trans-stimulation found with native microsomes, assays were done with microsomes in which the putative trans-stimulation compound(s) had been depleted by preincubating the vesicles for $40 \mathrm{~min}$ at $37^{\circ} \mathrm{C}$. Such pretreatment had only a minimal effect on intactness of the microsomal vesicles (latency of Man-6-phosphatase remained $>93 \%$ ).

Fig. $5 A$ shows that the translocation rate was saturable at UDPGlc substrate concentrations of 1-2 mM. The Scatchard representation of these data revealed complex kinetics (Fig. 5 $B$ ). A model incorporating two Michaelis-Menten kinetic components could be formulated that provided an excellent fit to the experimental results. The estimated values (from two experiments) for the kinetic parameters of these two components were 32-43 $\mu \mathrm{M}$ and 638-707 $\mu \mathrm{M}$ as apparent $K_{\mathrm{m}}$ and 53-70 pmol/min per $\mathrm{mg}$ protein and $298-348 \mathrm{pmol} / \mathrm{min}$ per $\mathrm{mg}$ protein as $V$ for, respectively, the high and low affinity components. The observed saturation suggests that a limited number of UDPGlc transport sites exist in the microsomal membrane. Even at high concentrations of radiolabeled UDPGlc substrate (at which a possible diffusion transport 
pathway would become important), alternative substrates were still effective inhibitors. For example, an eightfold excess of 5'-UMP over radiolabeled UDPGlc reduced the uptake rate of $25 \mu \mathrm{M}$ by $52 \%$ while translocation tested with a high, $2-\mathrm{mM}$ substrate concentration was reduced by $68 \%$. Based on these observations, it seems unlikely that the low affinity component corresponded to diffusion.

Subcellular localization of microsomal UDPGlc translocation. Because highly active nucleotide sugar transport systems have been identified in Golgi apparatus (22-28) and contamination of microsomal fractions with Golgi membranes is unavoidable $(29,30)$, evidence was sought to exclude the possibility that the carrier-mediated UDPGlc translocation found in our RER membrane preparations was solely dependent on Golgi structures. If Golgi but not ER membrane vesicles were responsible for transport, one would expect to find higher specific transport activities in the smooth microsomal fraction than in the RER microsomal preparation, which is considerably less contaminated with Golgi membranes (14). Thus, specific catalytic activities for the Golgi membrane marker ovomucoid:UDP-galactosyltransferase were 3.2- to 3.7-fold higher in our smooth microsomal fractions than in our RER membrane preparations. In contrast, specific UDPGlc translocation rates were clearly lower in smooth than in rough microsomes. In two separate subfractionation studies, UDPGlc transport rates measured with $25 \mu \mathrm{M}$ UDP $\left[{ }^{3} \mathrm{H}\right] \mathrm{Glc}$ were 10.8 and 10.3 $\mathrm{pmol} / \mathrm{mg}$ protein per min and 18.6 and $19.7 \mathrm{pmol} / \mathrm{mg}$ protein per min in, respectively, the smooth and rough subfractions. The difference was even larger $(79 \mathrm{pmol} / \mathrm{mg}$ per min in smooth microsomes vs. $305 \mathrm{pmol} / \mathrm{mg}$ per min in the RER fraction) when translocation rates were measured with $2 \mathrm{mM}$ UDP $\left[{ }^{3} \mathrm{H}\right] \mathrm{Glc}$ as substrate. Collectively, these results suggest that the postulated carrier system mediating entry of UDPGlc into the vesicles is present in ER membranes.

\section{Discussion}

The findings reported here provide strong evidence for the presence in ER-derived microsomes of a carrier system that specifically mediates translocation of intact UDPGlc from the cytosolic side of the membrane into the intramicrosomal space. Perez and Hirschberg (7) recently reported that UDPGlc is taken up by ER-derived vesicles and that this interaction involved specific binding or transport sites but failed to provide conclusive evidence for proper translocation of the intact nucleotide sugar across the membrane into the intravesicular space. In agreement with Perez and Hirschberg's work, we found that radionuclide binding by microsomes incubated with radiolabeled UDPGlc exhibited typical features of carrier-mediated uptake including marked temperature dependency (Fig. 2), saturation (Fig. 6), and substrate specificity (Table I). However, Perez and Hirschberg did not recognize the transient trans-stimulation of radiolabeled UDPGlc uptake caused by the presence of endogenous UDPGlc in native microsomes, which must be taken into consideration in kinetic studies of nucleotide sugar transport across the ER membrane. Whether the complex kinetics of microsomal UDPGlc uptake revealed in the present study, with what appears to be two Michaelis-Menten kinetic components, was a result of different expressions of the same carrier in the hetero-
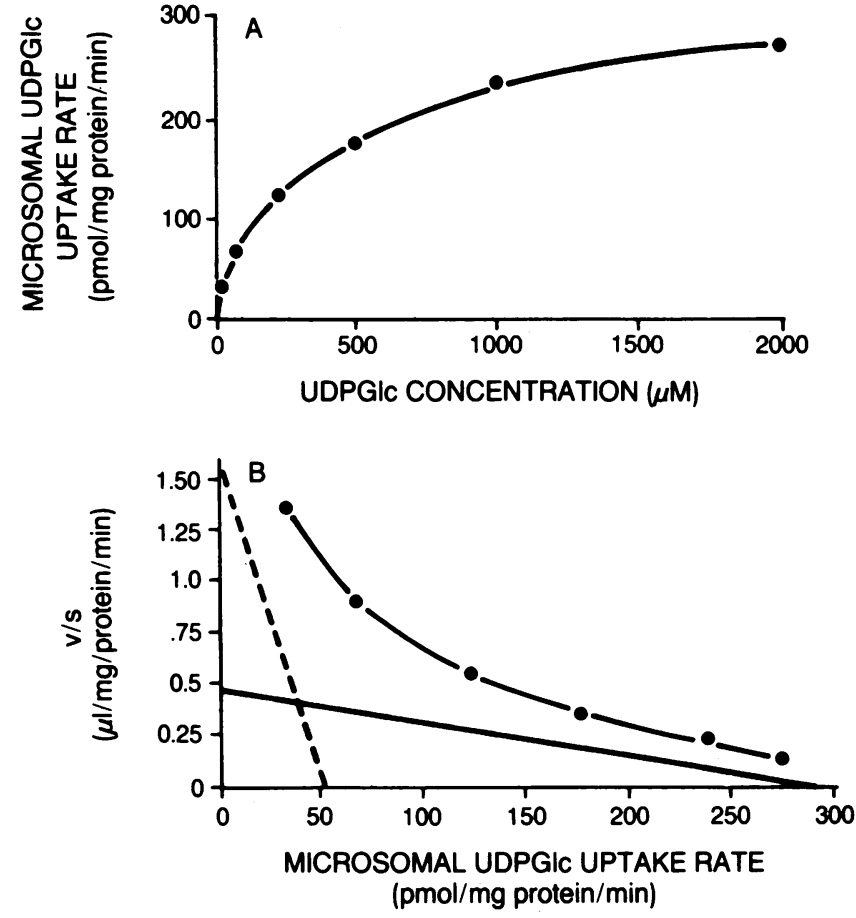

Figure 6. Dependence of rate of microsomal UDPGlc uptake on substrate concentration. Initial rate of microsomal uptake of radiolabel at UDP $\left[{ }^{3} \mathrm{H}\right] \mathrm{Glc}$ concentrations varying between $25 \mu \mathrm{M}$ and $2 \mathrm{mM}$ was assessed using RER microsomes that had been preincubated for 40 min at $37^{\circ} \mathrm{C}$ in buffer B. $(A)$ Rate of uptake of UDP $\left[{ }^{3} \mathrm{H}\right] \mathrm{Glc}$ equivalents plotted vs. substrate concentration. $(B)$ The same data in a Scatchard plot. Based on the assumption that the observed kinetics reflected involvement of two Michaelis-Menten kinetic components, a model could be formulated that yielded an excellent fit to the experimental results. The smooth curves in both graphs represent the fitted curves generated by this model. The straight lines drawn in $B$ depict the two individual Michaelis-Menten kinetic components. The estimates $( \pm \mathrm{SE})$ of the kinetic parameters for the experiment shown here were $K_{\mathrm{m}}$ values of $33 \pm 13 \mu \mathrm{M}$ and $0.64 \pm 0.14 \mathrm{mM}$ and $\mathrm{V}$ values of $53 \pm 17 \mathrm{pmol} / \mathrm{mg}$ per min for, respectively, the high-affinity (broken line) and low-affinity (solid line) components.

geneous vesicle structures of RER microsomal preparations remains to be elucidated. It is noteworthy in this regard that Meissner and Allen (17) noted similar heterogeneity of glucose transport properties in rat liver microsomes.

A persuasive argument in support of carrier-mediated transport of UDPGlc was derived from influx and efflux studies demonstrating that the trans concentration of the transported UDPGlc or presumed competitors for the postulated carrier system, including UMP, UDPGlcNAc, and UDPGlcUA, affected translocation of the labeled substrate (Figs. 4 and 5). Such counter-transport appeared to be responsible for the remarkable transient overshoot or trans-stimulation phenomenon observed in UMP and UDPGlc uptake studies using low concentrations of radiolabeled substrate and native microsomes that had not been preincubated at $37^{\circ} \mathrm{C}$.

A number of observations suggest that microsomal uptake of UDPGlc corresponded predominantly to proper translocation of the intact sugar nucleotide molecule into the interior space of the microsomal vesicles. We found that the apparent space occupied by vesicle-associated intact UDPGlc under 
equilibrium uptake conditions corresponded to an osmotically sensitive component, which was a strong indication for proper translocation into the intramicrosomal lumen. During the initial uptake phase, the radiolabel that became associated with the vesicles was mostly present in authentic UDPGlc. This was so at low as well as at high UDPGlc substrate concentrations and there clearly was no precursor-product relationship between any of the metabolic products of UDPGlc and the total amount of microsomal radiolabel. On the contrary, the time curves shown in Fig. 1 suggested that uptake of radiolabel preceded hydrolysis of the nucleotide sugar. Furthermore, neither free Glc and GlcUA nor their respective 1-monophosphate derivatives were able to inhibit microsomal uptake of radiolabeled UDPGlc indicating that the observed transport process did not depend on carrier-mediated transport of such hydrolytic fragments, Additional supportive evidence for translocation of the intact UDPGlc molecule across the membrane came from the observation that extravesicular UDPGlc, UMP, UDPGlcNAc, and UDPGlcUA were potent trans-stimulators of radiolabel efflux from microsomes preloaded with radiolabeled UDPGlc (Fig. $4 C$ ). In these experiments, microsomes first were preloaded by short preincubation with $25 \mu \mathrm{M}$ radiolabeled UDPGlc, i.e., conditions that produced the overshoot phenomenon for microsomal uptake of the nucleotide sugar. The radiolabel efflux measurements were started when overshooting peaked and $\sim 85 \%$ of the microsomal radiolabel was present in authentic UDPGlc (Fig. $1 A$ ). Hence, the abrupt and markedly accelerated efflux of radioactivity caused by the trans-stimulators could only occur if intact UDPGlc was translocated from the vesicles into the incubation medium. Moreover, acceleration of efflux of the other radiolabeled glucosyl-containing species, free Glc, Glc-1-P, or Dol-P-Glc, was not expected to occur as a result of addition of the trans-stimulators. Glc-1-P and free Glc did not seem to interact with the postulated UDPGlc carrier (see inhibition studies), and significant release of radiolabel from Dol-P-Glc, possibly by conversion to UDPGlc and Dol-P was unlikely, especially in the experiment showing trans-stimulation by $200 \mu \mathrm{M}$ unlabeled UDPGlc, which is a saturating substrate concentration for microsomal Dol-P-Glc synthase $(31,32)$. We cannot completely rule out that the observed translocation of UDPGlc reflects carrier-mediated transport of UMP, with reassembly of UDPGlc at the lumenal face of the ER membrane and secondary transport of compound(s) containing the radiolabeled Glc moiety derived from the UDPGlc substrate. However, several observations and considerations render such hypothesis implausible. For kinetic reasons (see above and reference 6), it is unlikely that the observed translocation of UDPGlc occurs via formation of a Dol-P-Glc intermediate. Resynthesis of UDPGlc from its hydrolysis products with reassembly of pyrophosphate ester bonds is unlikely from a thermodynamic viewpoint, considering the fact that the cytosolic synthesis of UDPGlc from Glc-1-P by pyrophosphorylase depends on the high energy compound UTP and resynthesis of UDPGlc would require effective conversion of UMP to UTP in the microsomes. This is unlikely, as is illustrated by the fact that microsomes incubated with UDPGlc (Fig, 1) or UTP (33) spontaneously hydrolyze these compounds, a reaction that runs to completion. Finally, it should be noted that the transstimulation experiments demonstrate that a high intramicrosomal concentration of UDPGlc trans-stimulates UDPGlc uptake. If UDPGlc uptake were driven by UMP transport and required resynthesis of UDPGlc within the microsomes, then one would expect that a high intramicrosomal UDPGlc concentration would inhibit, rather than stimulate, UDPGic uptake.

An additional important difference between our findings and the previous work by Perez and Hirschberg $(7,26)$ relates to the suggestion by the latter workers that both UDPGlc and UDPGlcNAc enter the microsomes by an antiport system exchanging lumenal UMP for external UDP-sugar. While such exchange was clearly observed, our data suggest that microsomal uptake of UDPGlc was not necessarily and exclusively coupled to UMP efflux and therefore they do not support the notion of an antiport translocation system. The observed exchange of UDPGlc for UMP reflected countertransport, as a result of dependency of translocation of both nucleotides on a shared carrier system. Thus, uptake of UDPGlc and UMP occurred in microsomes from which nucleotides had been "drained" by preincubation at $37^{\circ} \mathrm{C}$ (Fig. $4 \mathrm{~A}$ ) and UDPGlc efflux was markedly stimulated by the presence of UDPGlc itself or UDPGICNAc or UDPGIcUA in the extravesicular medium (Fig. $4 C$ ). Similarly, UMP in the extramicrosomal incubation medium was a trans-stimulator of efflux of UMP from the vesicles (data not shown), indicating that UMP translocation in either direction across the membrane occurred without necessity for coupling to nucleotide sugar transport.

Our findings on substrate specificity of the postulated microsomal carrier system for UDPGlc are in general agreement with the results of Perez and Hirschberg (7), except for the binding of UDPGal to the carrier. We found that this nucleotide sugar, like the other tested $\dot{5}^{\prime}$-uridine-substituted nucleotides, was an effective cis-inhibitor of UDPGlc translocation by RER-derived microsomes, suggesting that UDPGal might be a substrate of the postulated UDPGlc carrier in ER membranes. Perez and Hirschberg on the other hand found that UDPGal (tested at $20 \mu \mathrm{M}$ ) was a poor trans-stimulator of UMP efflux from RER microsomes (7) and observed almost no uptake of radiolabel when RER-derived yesicles were incubated with $2 \mu \mathrm{M}$ UDP $\left[{ }^{3} \mathrm{H}\right] \mathrm{Gal}$ while at the same substrate concentration rapid uptake of the nucleotide sugar occurred with Golgi membrane preparations (26). Translocation of UDPGal by RER- or Golgi-derived vesicles has not been directly examined in the present study. A possible explanation for the apparent discrepancy could be that the UDPGlc carrier system of ER membranes may have a low affinity for UDPGal so that an effect of UDPGal on translocation of UBPGlc or UMP only became detectable at the high UDPGal concentration $(200 \mu \mathrm{M})$ used in our study and uptake of,UDPGal itself by RER microsomes remained small at the very low ( 2 $\mu M)$ substrate concentration used in Perez and Hirsthberg's studies.

The finding that UDPGlcUA was an efficient cis-inhibitor of microsomal UDPGlc uptake and trans-stimulator of UDPGlc efflux from the vesicles is of special interest in view of the importance of UDPGlcUA as a cosubstrate for microsomal glucuronidation reactions. It has been suggested that the marked latency of transferase activity characterizing all isoforms of UDP-glucuronyltransferase in native microsomes may result from lumenal orientation of the enzyme $(2,34)$. Such topology would necessitate permeation of UDPGlcUA 
from cytosol, where its synthesis occurs, across the microsomal membrane to a luminally oriented catalytic center. Whether such topological arrangement of the enzyme exists and transport of UDPGlcUA across the membrane is rate-limiting for glucuronidation by native microsomes and thereby explains the latency phenomenon remains unresolved. Our results suggest that UDPGIcUA specifically interacts with the postulated carrier for UDPGlc in ER membranes. The observation that UDPGlcUA trans-stimulated efflux of UDPGlc from the microsomes (Fig. $4 C$ ) especially suggested that UDPGIcUA undergoes transport into the vesicles because the trans-stimulation phenomenon implies that binding of the "driver" UDPGlcUA solute to carrier at the cytosolic side of the membrane produces translocation of the complex to the luminal side and thereby leads to enhanced access of the "driven" solute present in the intravesicular space (in this case radiolabeled UDPGlc) to the carrier's transport sites. Preliminary experiments suggest that the RER-derived vesicles can also take up radiolabeled UDPGlcUA and that such microsomal uptake was $c i s$-inhibited by UDPGlc and displayed marked temperature dependency. However, only a negligible proportion of the vesicle-associated radiolabel was detectable in intact UDPGlcUA and a major fraction of the microsomal radioactivity was TCA-precipitable, suggesting that either internalized UDPGlcUA was instantaneously and extensively metabolized or microsomal uptake reflected only binding and metabolism to membrane-linked derivatives, without proper translocation across the membrane (our unpublished results). While the question whether UDPGlcUA can be translocated across the ER membrane remains unresolved, it is worth noting that our inability in these preliminary experiments to detect intact UDPGlcUA in the vesicle-associated radiolabel was in agreement with the apparent absence of a microsomal pool of endogenous UDPGlcUA in native, intact microsomes (7).

\section{Acknowledgments}

The authors are indebted to Drs. V. Licko, B. Scharschmidt, R. Van Dyke, and D. Warnock for helpful discussions, to Dr. M. Barker for electron microscopy examination of subcellular fractions, to Mr. Kong Pua for excellent technical assistance, and to Diana Fedorchak and Michael Karasik for outstanding editorial assistance.

This work was supported by grants AM-11275 and AM-26732 from the National Institutes of Health and by National Institutes of Health International Research Fellowship and North Atlantic Treaty Organization Research Fellowship awards to Dr. Vanstapel.

\section{References}

1. Dutton, G. J. 1980. Glucuronidation of Drugs and Other Compounds. CRC Press, Inc., Boca Raton, FL.

2. Hallinan, T., and A. E. R. de Brito. 1981. Topology of endoplasmic reticular enzyme systems and its possible regulatory significance. In Hormones and Cell Regulation. Vol. 5. J. E. Dumont, and J. Nunez, editors. Elsevier/North Holland, Amsterdam. 73-95.

3. Kornfeld, R., and S. Kornfeld. 1985. Assembly of asparaginelinked oligosaccharides. Annu. Rev. Biochem. 54:631-664.

4. Zakim, D., and D. A. Vessey. 1976. The effects of lipid-protein interactions on the kinetic parameters of microsomal UDP-glucuronyltransferase. In Membranes. Vol. 2. A. I. Martonosi, editor. Plenum Press, New York. 443-461.

5. Hochman, Y., M. Kelley, and D. Zakim. 1983. Modulation of the number of ligand binding sites of UDP-glucuronyltransferase by the gel to liquid crystal phase transition of phosphatidylcholines. $J$. Biol. Chem. 258:6509-6516.

6. Vanstapel, F., and N. Blanckaert. 1987. Endogenous esterification of bilirubin by liver microsomes. Evidence for an intramicrosomal pool of UDP-glucose and luminal orientation of bilirubin UDP-glycosyltransferase. J. Biol. Chem. 262:4616-4623.

7. Perez, M., and C. B. Hirschberg. 1986. Topography of glycosylation reactions in the rough endoplasmic reticulum membrane. J. Biol. Chem. 261:6822-6830.

8. Markwell, M. A. K., S. M. Haas, L. L. Bieber, and N. E. Tolbert. A modification of the Lowry procedure to simplify protein determination in membrane and lipoprotein samples. Anal. Biochem. 87:206210.

9. Vanstapel, F., K. Pua, and N. Blanckaert. 1986. Assay of mannose-6-phosphatase in untreated and detergent-disrupted rat liver microsomes for assessment of integrity of microsomal preparations. Eur. J. Biochem. 156:73-77.

10. Schneider, W. C. 1957. Determination of nucleic acids in tissues by pentose analysis. Methods Enzymol. 3:680-684.

11. Bretz, R., and W. Staubli. 1977. Detergent influence on ratliver galactosyltransferase activities towards different acceptors. Eur. J. Biochem. 77:181-192.

12. Masters, B. B., S., C. H. Williams, Jr., and K. Kamin. 1967. The preparation and properties of microsomal TPNH-cytochrome c reductase from pig liver. Methods Enzymol. 10:565-567.

13. Leyten, R., J. P. A. M. Vroemen, N. Blanckaert, and K. P. M. Heirwegh. 1980. The congenic normal R/APfd-j/j rat strains. A new animal model of hereditary nonhemolytic unconjugated hyperbilirubinemia due to nonhemolytic unconjugated hyperbilirubinemia due to defective bilirubin conjugation. Lab. Animals. 20:335-342.

14. Eriksson, L. C., J. W. DePierre, and G. Dallner. 1978. Preparation and properties of microsomal fractions. Pharmacol. Ther. A. 2:281-317.

15. Arion, W. J., L. M. Ballas, A. J. Lange, and B. K. Wallin. 1976. Microsomal membrane permeability and the hepatic glucose-6-phosphatase system. Interactions of the system with D-mannose 6-phosphate and D-mannose. J. Biol. Chem. 251:4901-4907.

16. Nillson, R., E. Peterson, and G. Dallner. 1973. Permeability of microsomal membranes isolated from rat liver. J. Cell Biol. 56:762776.

17. Meissner, G., and R. Allen. 1981. Evidence for two types of rat liver microsomes with differing permeability to glucose and other small molecules. J. Biol. Chem. 256:6413-6422.

18. Faltynek, C. R., J. E. Silbert, and L. Hof. 1981. Inhibition of the action of pyrophosphatase on sugar nucleotides. J. Biol. Chem. 256:7139-7141.

19. Godelaine, D., H. Beaufay, and M. Wibo. 1977. Incorporation of $\mathrm{N}$-acetylglucosamine into endogenous acceptors of rough microsomes from rat liver: stimulation by GTP after treatment with pyrophosphate. Proc. Natl. Acad. Sci. USA. 74:1095-1099.

20. Bansal, S. K., and T. Gessner. 1980. A unified model for the assay of uridine diphosphoglucuronyltransferase activities toward various aglycones using uridine diphospho $\left[\mathrm{U}-{ }^{14} \mathrm{C}\right]$ glucuronic acid. Anal. Biochem. 109:321-329.

21. Fleischer, B. 1981. The nucleotide content of rat liver Golgi vesicles. Arch. Biochem. Biophys. 212:602-610.

22. Kuhn, N. J., and A. White. 1976. Evidence for specific transport of uridine diphosphate galactose across the Golgi membrane of rat liver mammary gland. Biochem. J. 154:243-244.

23. Carey, D. J., L. W. Sommers, and C. B. Hirschberg. 1980. CMP-N-acetyl-neuraminic acid: isolation from and penetration into mouse liver microsomes. Cell. 19:597-605.

24. Sommers, L. W., and C. B. Hirschberg. 1982. Transport of sugar nucleotides into rat liver Golgi. A new Golgi marker activity. $J$. Biol. Chem. 257:10811-10817.

25. Capasso, J. M., and C. B. Hirschberg. 1984. Mechanisms of glycosylation and sulfation in the Golgi apparatus: evidence for nu- 
cleotide sugar/nucleoside monophosphate and nucleoside sulfate/nucleoside monophosphate antiports in the Golgi apparatus membrane. Proc. Natl. Acad. Sci. USA. 81:7051-7055.

26. Perez, M., and C. B. Hirschberg. 1985. Translocation of UDP-N-acetyl-glucosamine into vesicles derived from rat liver rough endoplasmic reticulum and Golgi apparatus. J. Biol. Chem. 260:4671-4678.

27. Fleischer, B. 1983. Mechanism of glycosylation in the Golgi apparatus. J. Histochem. Cytochem. 31:1033-1040.

28. Barthelson, R., and S. Roth. 1985. Topology of UDP-galactose cleavage in relation to $\mathrm{N}$-acetyl-lactosamine formation in Golgi vesicles. Biochem. J. 225:67-75.

29. Fleischer, S., and M. Kervina. 1974. Subcellular fractionation of rat liver. Methods Enzymol. 31:6-41.

30. Eriksson, L. C., A. Bergstrand, and G. Dallner. 1977. Lateral topology in the rough endoplasmic reticulum of rat liver. Cell Tissue Res. 179:17-43.

31. Scher, M. B., W. A. Burton, and C. J. Waechter. 1980. Enzymatic glucosylation of dolichol monophosphate formed via cytidine triphosphate in calf brain membranes. J. Neurochem. 35:844-849.

32. Ravoet, A.-M., A. Amar-Costesec, D. Godelaine, and H. Beaufay. 1981. Quantitative assay and subcellular distribution of enzymes acting on dolichol phosphate in rat liver. J. Cell Biol. 91:679-688.

33. Finch, S. A. E., T. F. Slater, and A. Stier. 1979. Nucleotide metabolism by microsomal UDP-glucuronyltransferase and nucleoside diphosphatase as determined by ${ }^{31} \mathrm{P}$ nuclear-magnetic-resonance spectroscopy. Biochem. J. 177:925-930.

34. Mackenzie, P. I. 1986. Rat liver UDP-glucuronyltransferase. Sequence and expression of a cDNA encoding a phenobarbital-inducible form. J. Biol. Chem. 261:6119-6125. 